\title{
Some aspects of the three-dimensional double-diffusive natural convection in a parallelepipedic tilted solar distiller
}

\author{
Fakher OUESLATI, Brahim BEN-BEYA, Taieb LILI
}

Faculty of Sciences of Tunis, Laboratory of Mechanic of Fluids, Physics Department, University of Tunis El-Manar, 2092, Tunis, Tunisia

E-mail adresses: fakher.oueslati@gmail.com;

Brahim.benbeya@fst.rnu.tn; taieb.lili@fst.rnu.tn

Keywords: Solar distiller; double diffusive natural convection; three-dimensional flow, inclined enclosure.

\begin{abstract}
Three-dimensional double-diffusive natural convection in a parallelepipedic solar distiller inclined with an angle is investigated in the current study. Computations are performed using a home code "NASIM" based on the finite volume method and a full multigrid technique. It is found that iso-surfaces relative to temperature field undergo a central stratification while the lower and upper gradients seem to be significantly strengthened by gradually increasing the Rayleigh number values. In terms of buoyancy ratio effects, projection of thermal and solutal isocontours at the mid plane $(y=1)$ showed that the flow intensity is significantly enhanced by monotonously increasing $\mathrm{N}$ for aiding flow situation $(\mathrm{N}>0)$. In addition, and according to all Rayleigh number values, the variation of average Nusselt and Sherwood numbers seem to be minimum for $\mathrm{N}=-1$ with weaker values for opposing flow situation. On another hand, isosurfaces of the transverse v-velocity component showed the importance of the 3-D effects that manifest within the solar distiller.
\end{abstract}

\section{INTRODUCTION}

Thermosolutal natural convection has received increasing research attention during the past few decades. In nature, it extensively exists in oceanography, geophysics and geology. In fact, there are many important applications in engineering, such as for energy storage systems, solar engineering and some material processing. For instance, many studies on the heat and mass transfer phenomena primarily focused on analyzing the heat and mass phenomena in two-dimensional cavities [1, 2]. However, only a few studies focused on the doublediffusive convection within three-dimensional enclosures flow. In this context, Sezai and Mohamad [3] numerically examined the double diffusive convection within a cubic enclosure and indicated that the thermosolutal flow in enclosures with opposing buoyancy forces is strictly three dimensional for a certain range of parameters. Very recently, unsteady three-dimensional (3D) double diffusive convection in tilted enclosure having a parallelepipedic shape has been analyzed numerically by Oueslati et al. [4]. It was found that the optimal heat and mass transfer rates for the aiding situation have been observed at two particular inclination angles $\gamma$; namely $30^{\circ}$ and $75^{\circ}$ about the horizontal direction. In addition, the flow was observed to undergo a periodic behavior for the particular parameters $\mathrm{Ra}=10^{4}$ and an inclination angle $=75^{\circ}$ according to the aiding flow case.

On another hand, one of the main industrial applications of double diffusive natural convection is solar distillation in which natural convection occurs within the solar distiller, owing to the collective thermal and mass diffusion buoyancy effects as well as the temperature difference between a cover and an absorber [5-7]. In fact, study of solar distillers is one of the most significant works to progress their performance with a competitive price Madhlopa et al. 
[8]. In this vein, it is known that numerical investigation allows testing the performance of solar distillers for different configurations and parameters. Besides, numerical study has the advantage over an experimental investigation in that the important parameters, such as glass thickness, geometrical dimensions, and covenant location. However, until now, only a few numerical investigations of the heat and mass transfer in solar distillers have been presented, especially for inclined enclosure. In fact, the three-dimensional transverse flow is primordial when dealing with the intensification of heat and mass transfers in a solar distiller, and it is still not extensively studied in literature. Chouikh et al. [9] performed a numerical study concerning the 2-D inclined solar distiller flow for an aspect ratio 10. These authors have found that the enhanced heat and mass transfer rates are obtained for low Rayleigh numbers. Besides, the average Nusselt number value increases slightly with increasing inclination angle from $0^{\mathrm{O}}$ to about $40^{\circ}$, and then, it decreases steeply with increasing inclination angle until about $40^{\circ}$. Another numerical study on optimization of a solar distiller dimensions has been performed by Omri [10]. He pointed out that the geometry of the solar distiller is of a great importance in predicting the adequate dimensions and covenant location of vapor production and condensations within the solar distiller. Not long ago, Rahman et al. [11] conducted a mathematical study for double diffusive natural convection flow inside a two-dimensional triangular shaped solar collector. They found that both heat transfer and mass transfer increase with increasing Rayleigh number. Furthermore symmetric flow field temperature distribution and mass distribution are found according to the middle axis of the triangular cavity.

On another hand, an extension of the present work by studying the effect the inclination previously showed that a minimum of the heat and mass transfer rates are obtained for an angle value of $75^{\circ}$ for the aiding case. From this point of view, the aim of the current study is to predict and discuss the three-dimensional double diffusive convection flow within a parallelepipedic solar distiller tilted at an angle with respect to the horizontal line. The 3D flow characteristics and thermosolutal fields are analyzed in terms of velocity isocontours, temperature iso-surfaces, isotherms and iso-concentration at the mid-plane $(y=1)$, and average heat and mass transfer rates. Particular attention is also attributed to 3-D effects of the transverse direction.

\section{PHYSICAL MODEL AND METHOD OF SOLUTIONS}

Details of the geometry for the configuration are shown by a schematic diagram in Fig. 1. The physical model considered here is a three-dimensional solar distiller tilted with an inclination angle. The enclosure is a parallelepiped of width $\mathrm{W}$, height $\mathrm{H}$ and depth $\mathrm{D}$ with aspect ratios $\mathrm{Ay}=\mathrm{D} / \mathrm{W}=2$ and $\mathrm{Az}=\mathrm{Ax}=\mathrm{H} / \mathrm{W}=1$.

By employing the aforementioned assumptions, the conservation equations of mass, momentum, energy and species can be expressed in their dimensionless forms as follows: 


$$
\begin{aligned}
& \frac{\partial u}{\partial x}+\frac{\partial v}{\partial y}+\frac{\partial w}{\partial z}=0 \\
& \frac{\partial u}{\partial t}+\frac{u \partial u}{\partial x}+\frac{v \partial u}{\partial y}+w \frac{\partial u}{\partial z}=\square \frac{\partial p}{\partial x}+\operatorname{Pr}\left(\frac{\partial^{2} u}{\partial x^{2}}+\frac{\partial^{2} u}{\partial y^{2}}+\frac{\partial^{2} u}{\partial z^{2}}\right)+\operatorname{Ra} \operatorname{Pr}(\theta+N c) \sin \gamma \\
& \left.-+\frac{\partial v}{\left.\partial^{2} v\right) \partial t}+\frac{u \partial v}{\partial t}+w \frac{v \partial v}{\partial y}=\square \frac{\partial v}{\partial z}+\operatorname{Pr} \frac{\partial p}{\partial y}+\frac{\left(\partial^{2} v\right.}{\left(\partial x^{2}\right.}+\frac{\partial^{2} v}{\partial y^{2}}\right) \\
& \frac{\partial w}{t}+\frac{\partial \partial w}{x}+\frac{\partial \partial w}{y}+w \frac{\partial w}{z}=\square \frac{\partial p}{z}+\operatorname{Pr}\left(\frac{\partial^{2} w}{\partial x^{2}}+\frac{\partial^{2} w}{\partial y^{2}}+\frac{\partial^{2} w}{\partial z^{2}}\right)+\operatorname{RaPr}(\theta+N c) \cos \gamma \\
& \frac{\partial \theta}{\partial t}+\frac{u \partial \theta}{\partial x}+\frac{v \partial \theta}{\partial y}+\frac{w \partial \theta}{\partial z}=\left(\frac{\partial^{2} \theta}{\partial x^{2}}+\frac{\partial^{2} \theta}{\partial y^{2}}+\frac{\partial^{2} \theta}{\partial z^{2}}\right) \\
& +\frac{\partial c}{\partial t}+\frac{u \partial c}{\partial x} \quad \frac{v \partial c}{\partial y}+\frac{w \partial c}{\partial z} \quad \frac{1}{L e}\left(\frac{\partial^{2} c}{\partial x^{2}} \frac{\partial^{2} c}{\partial y^{2}} \frac{\partial^{2} c}{\partial z^{2}}\right)
\end{aligned}
$$

The dimensionless quantities $\mathrm{xi}_{\mathrm{i}}=(\mathrm{x}, \mathrm{y}, \mathrm{z}), \mathrm{u}_{\mathrm{i}}=(\mathrm{u}, \mathrm{v}, \mathrm{w}), \mathrm{t}, \mathrm{p}, \theta, \mathrm{c}$ denote the coordinates space, velocity component in the $\mathrm{xi}$ direction, time, hydrodynamic pressure, temperature, and concentration, respectively.

The governing equations (2-5) are non-dimensionalized using scales $W, \alpha / W, W^{2} / \alpha, \rho_{0} \alpha^{2}$ $/ W^{2}, \Delta T, \Delta C$ for coordinate space, velocity, time, pressure, temperature, concentration, respectively, where the characteristics of temperature and concentration scales $\Delta T$ and $\Delta C$ are defined as: $\Delta T=T_{h} \quad T_{c}$ and $\Delta C=C_{h} \quad C_{c}$.

The dimensionless temperature $\theta$ and concentration $\mathrm{c}$ are defined as $\theta=(\mathrm{T}-\mathrm{T} 0) / \Delta \mathrm{T}$ and $\mathrm{c}=(\mathrm{C}$ $\left.\mathrm{C}_{0}\right) / \Delta \mathrm{C}$.

Herev is the kinematic viscosity of the fluid, $\alpha$ and $D$ the thermal and mass diffusivities, respectively, and $\mathrm{g}$ is the acceleration due to gravity. Schmidt number can also be introduced as $S c=P r L e$. For appropriate boundary conditions, non-slip boundary conditions are imposed over the walls $(\mathrm{u}=\mathrm{v}=\mathrm{w}=0)$. Thermal and solutal boundary conditions are as follows: 
At $\mathrm{x}=0: \theta=\mathrm{c}=1$,

At $\mathrm{x}=1: \theta=\mathrm{c}=0$,

Elsewhere : $\frac{\partial \theta}{\partial n}=\frac{\partial c}{\partial n}=0$, where $\mathrm{n}$ is the sur:

coordinate.

The local Nusselt and Sherwood numbers are

$$
N u(x)=\left.\square \frac{\partial \theta}{\partial y}\right|_{x=0,1} \text { and } \operatorname{Sh}(x)=\left.\square \frac{\partial c}{\partial y}\right|_{x=0,1},
$$

the average Nusselt and Sherwood numbers as

$$
\begin{aligned}
& \overline{N u}=\frac{1}{\mathrm{~A}_{\mathrm{y}} \mathrm{A}_{\mathrm{z}}} \int_{0}^{\mathrm{A}_{\mathrm{y}} \mathrm{A}_{z}} \int_{0} N u(x) \mathrm{dyd} z \\
& \overline{S h}=\frac{1}{\mathrm{~A}_{\mathrm{y}} \mathrm{A}_{\mathrm{z}}} \int_{0}^{\mathrm{A}_{\mathrm{y}} \mathrm{A}_{z}} \int_{0} S h(x) \mathrm{dyd} \mathrm{z}
\end{aligned}
$$

It is worth noting that simulations were performed with a grid $32 \times 64 \times 32$ by using a developed home code named "NASIM" Ben-Beya and Lili [13] and the following numerical methodology. The temporal discretization of the time derivative is performed by an Euler backward second-order implicit scheme. The strong velocity-pressure coupling present in the continuity and the momentum equations is handled by implementing the projection method Brown et al. [14]. The Poisson equation which is solved using an accelerated full multigrid method Ben-Cheikh et al. [15], while the discretized equations are computed using the red and black point successive over-relaxation method Barrett et al. [16] with the choice of optimum relaxation factors. Finally, the convergence of the numerical results is established at each time step according to the following criterion:

$$
\sum_{i, i, k}\left|\Phi_{i, j, k}^{n} \square \Phi_{i, j, k}^{n \square 1}\right|<10^{\square 8}
$$

The generic variable $\Phi$ stands for $u, v, w, p, \theta$ or $\mathrm{c}$ and, $\mathrm{n}$ indicates the iteration time levels. In the above inequality, the subscript sequence $(\mathrm{i}, \mathrm{j}, \mathrm{k})$ represents the space coordinates $x, y$ and $z$. Because of the presence of large gradients near the walls, we generate a centro-symmetric grid with clustering near the walls using the following grid point distribution: 
$x_{i}=\frac{1}{2} 1+\left(\frac{\tanh (2 i / n \square 1)}{\tanh \xi}\right)$

where $\xi=1.25$ and $1 \leq \mathrm{i} \leq n$. Similar grid point distribution has been used in the three directions of the cavity.

\section{RESULTS AND DISCUSSION}

A. Effects of Rayleigh number
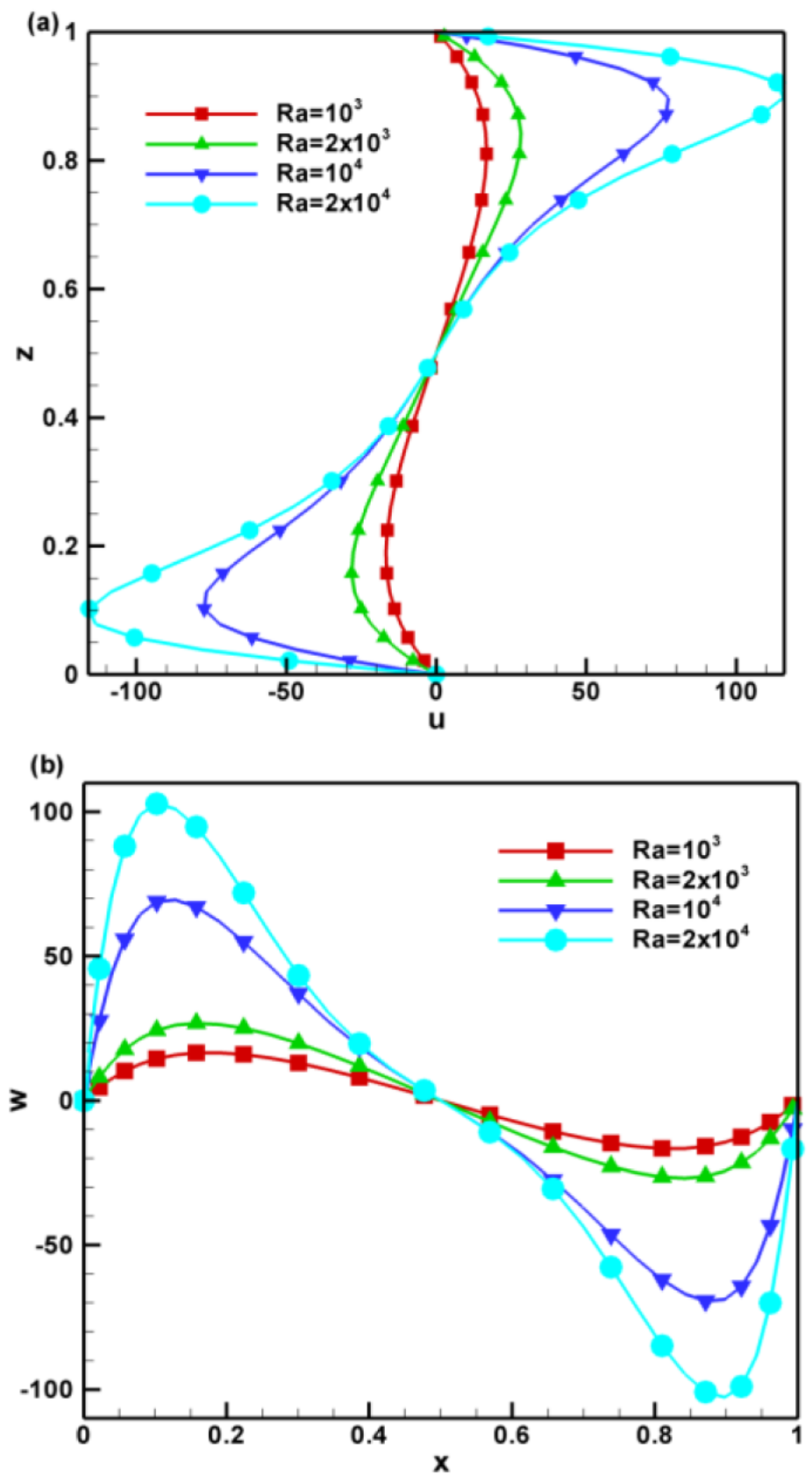

Fig. 2. Velocity component profiles $\mathrm{u}(\mathrm{z})$ and $\mathrm{w}(\mathrm{x})$ at the centerline $(\mathrm{y}=1)$ which correspond to $\mathrm{Ra}=10^{3}, 2.10^{3}, 10^{4}$ and $2.10^{4}$ for the buoyancy ratio value $\mathrm{N}=5$ : (a) $\mathrm{u}(\mathrm{z})$ and (b) $\mathrm{w}(\mathrm{x})$. 
The numerical results were performed for $\mathrm{Pr}=0.71, \mathrm{Le}=0.85$ and $\mathrm{Sc}=0.6035$ which cover water vapor diffusion into air. It is worth noting that all simulations were calculated for Rayleigh number values in the range $10^{3} \leq R a \leq 2 \times 10^{4}$. Furthermore, the buoyancy ratio $\mathrm{N}$ is varied in the range $(-5,5)$. In this section we attempt to analyze the influence of the Rayleigh number on threedimensional flow patterns within the solar distiller.

Figs. 2 (a) and (b) illustrates the horizontal and vertical velocity component distributions of $u$ $(z)$ and $w(x)$ at the mid-plane $(y=1)$, for $\mathrm{Ra}=10^{3}, 2.10^{3}, 10^{4}$ and $2.10^{4}$ and with respect to the buoyancy ratio value $\mathrm{N}=5$. As the velocity distribution indicates, the boundary layer is more closely confined to the walls with increase in the Rayleigh number. Moreover, both velocity profiles present a perfect symmetry about the center of the cavity. It is also observed that the velocity norm increases with the Rayleigh number meaning that thermosolutal natural convection dominates at high Ra.
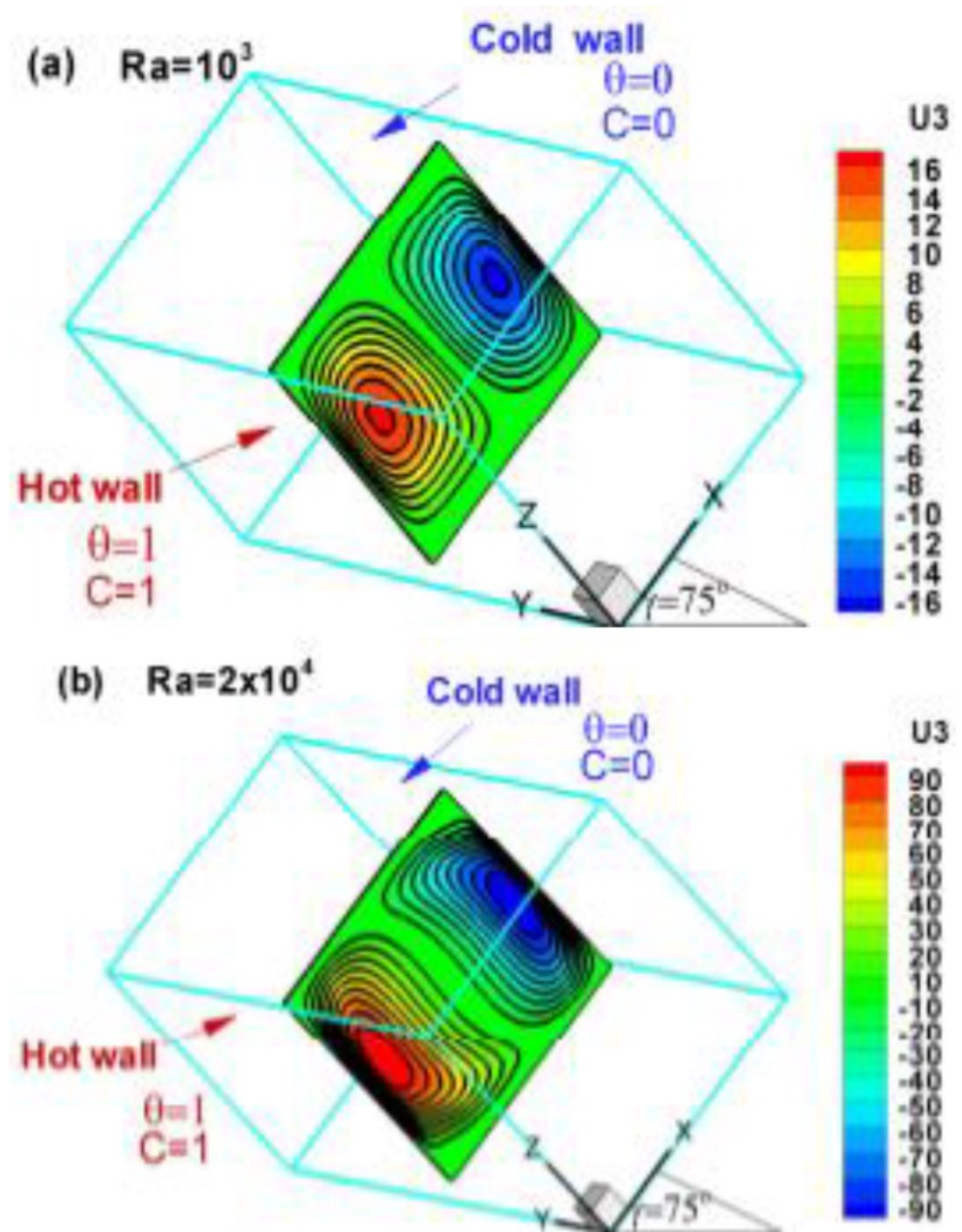

Fig. 3 Comparison of $\mathrm{w}$-isocontours for $\mathrm{Ra}=10^{3}$ and $2.10^{4}$ at the mid-plane $(\mathrm{y}=1)$ for a fixed buoyancy ratio value $\mathrm{N}=5$ : (a) $\mathrm{Ra}=10^{3}$ and (b) $\mathrm{Ra}=2.10^{4}$.

Isocontours relative to $\mathrm{w}$-velocity components related to $\mathrm{Ra}=10^{3}$ and $2.10^{4}$ are reported in Figs. 3(a) and (b), respectively. For lower Rayleigh number $10^{3}$, the flow is described by two counter rotating cells symmetrically distributed by the mid-plane $(x=0.5)$. By 
increasing Ra up to the value $2.10^{4}$, the two vortices seem to exhibit a great distortion and to approach the active walls while the core of the cavity is expected to remain at rest.

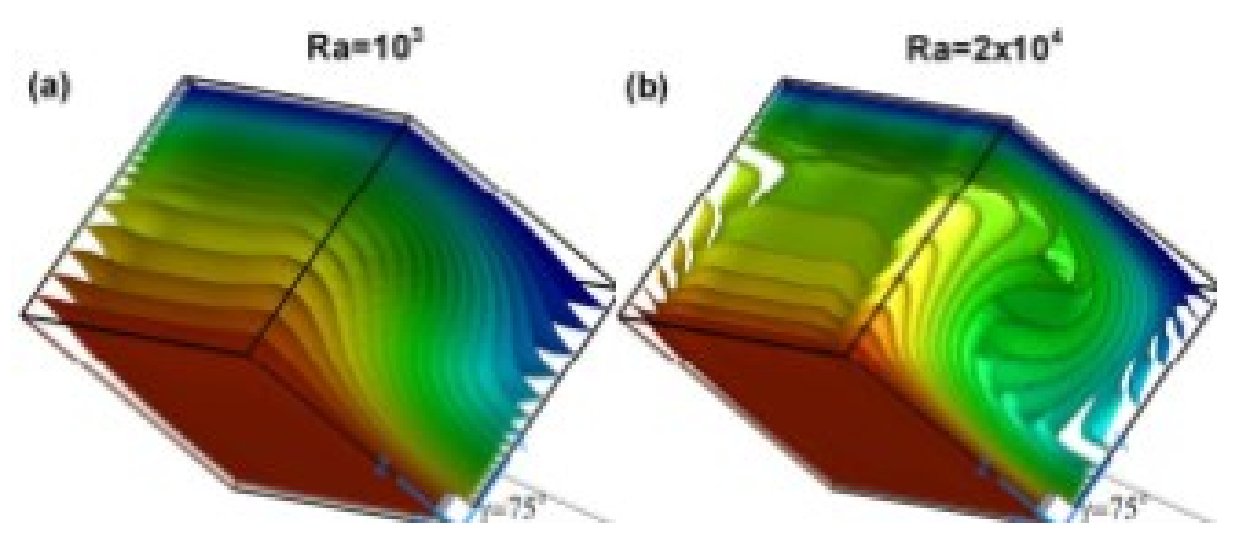

Fig. 4 Isosurfaces of the temperature for $\mathrm{Ra}=10^{3}$ and $2.10^{4}$ for a fixed buoyancy ratio value $\mathrm{N}=5$ : (a) $\mathrm{Ra}=10^{3}$ and (b) $\mathrm{Ra}=2.10^{4}$

At Fig. 4 are depicted a comparison of the temperature isosurfaces corresponding to two Rayleigh number values $R a=10^{3}$ and $R a=2 \times 10^{4}$ at $\mathrm{N}=5$ which is the maximum investigated buoyancy ratio value in this work. As depicted in the corresponding figure, for $R a=10^{3}$, the isotherms present slight distortion at the core of the enclosure with excessive gradients stratified near the lower part of the hot wall and the upper part of the cold wall. By augmenting Ra values to $R a=2 \times 10$, a central stratification clearly appears while the lower and upper gradients seem to be significantly strengthened. Consequently, one can obviously deduce that thermosolutal convection is augmented by monotonously increasing the Rayleigh number value. It is to notice that a similar pattern has been observed according to concentration fields, and we have chosen to only present temperature isosurfaces for brevity. 
B. Influence of buoyancy ratio and $3 D$ effects
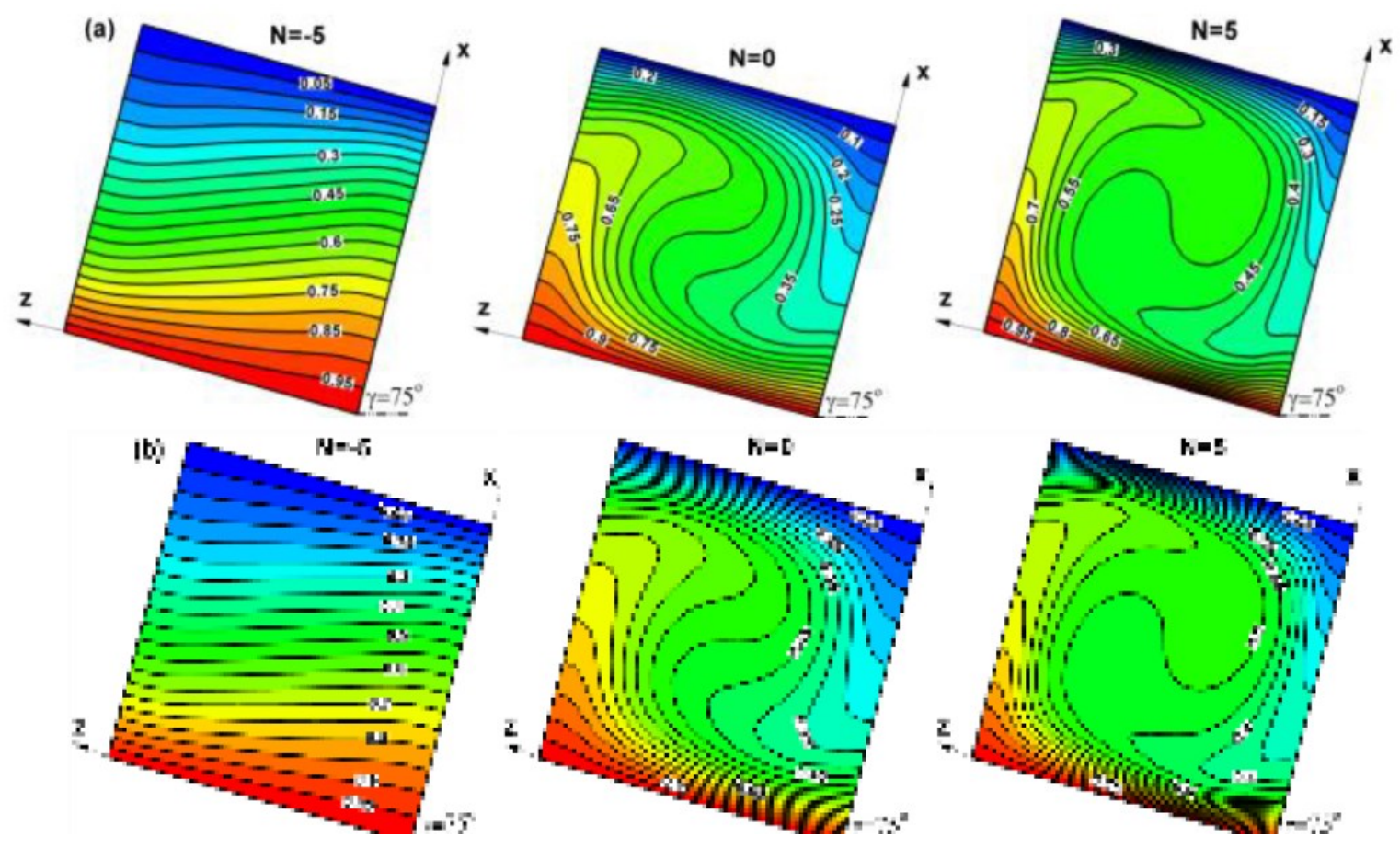

Fig. 5. Flow patterns for $\mathrm{Ra}=2 \cdot 10^{4}$ at the mid-plane $(\mathrm{y}=1)$, and different buoyancy ratio $\mathrm{N}=-5,0$ and 5: (a) isotherms, and (b) concentration isocontours.

In order to predict the influence of the buoyancy ratio $\mathrm{N}$ on the flow structure within the solar distiller, projections of thermal and solutal isocontours at the mid-plan $(\mathrm{y}=1)$ are illustrated at Figs. 5 (a) and (b), for $\mathrm{N}=-5,0$ and 5, respectively. As witnessed, a similar behavior is observed for both isotherms and iso-concentrations within the center of the cavity. For the opposing flow situation corresponding to $\mathrm{N}=-5$, the contour lines seem to be nearly parallel meaning that the double diffusive convection is quite weak. However, for aiding flow case $(\mathrm{N}>0)$, the flow intensity increases accordingly. In fact, at the limit situation $(\mathrm{N}=0)$, a distortion of the isotherms and isocontours is observed within the center core of the solar distiller. Meanwhile, isotherms and iso-contours are observed to be parallel and compressed at the vicinity of the active walls, thereby resulting in an acceleration of vertical boundary layer at these regions. Furthermore, when the flow is compositionally dominated $(\mathrm{N}=5)$, the central distortion is significantly enhanced. 

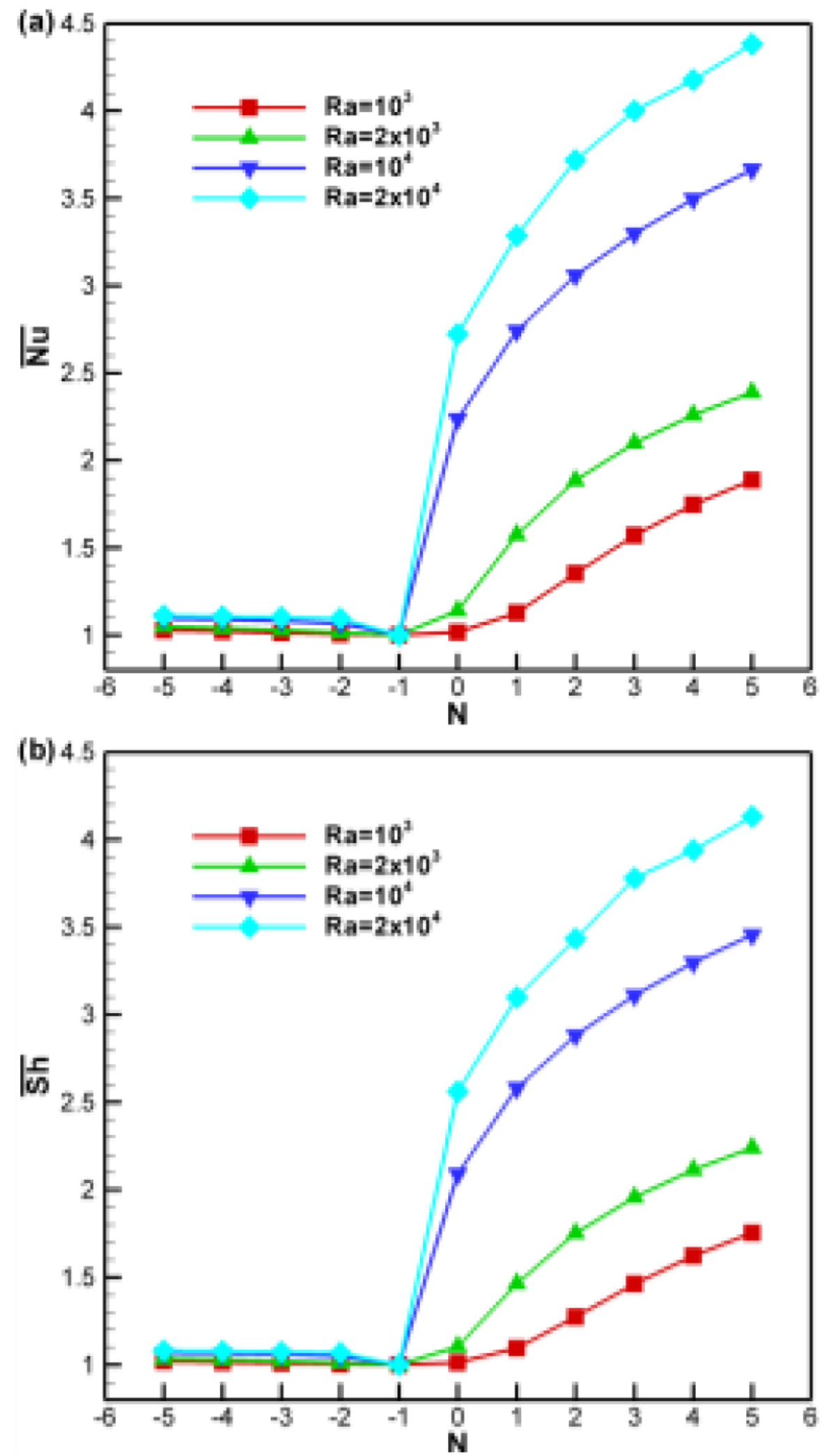

Fig. 6. Combined effects of Rayleigh number $\mathrm{Ra}$ and buoyancy ratio $\mathrm{N}$ on heat and mass transfer rates for $\mathrm{N}=5$, and with different buoyancy values $(-5 \leq \mathrm{N} \leq 5)$ : (a) average Nusselt number, and (b) average Sherwood number.

In order to further demonstrate the effect of buoyancy ratio $\mathrm{N}$ on the heat and mass transfer rates within the solar distiller, variations of both average Nusselt and Sherwood numbers with respect to $\mathrm{N}$ and for different Rayleigh number values are depicted in Fig.6. A first examination of the profile trends relative to opposing flow shows that average Nusselt and Sherwood 
values remain almost constant for $\mathrm{N}<-1$, while the corresponding profiles seem to undergo an augmenting behavior thereafter. In fact, and according to aiding flow situation, the heat and mass transfer rates are clearly enhanced by gradually increasing the buoyancy values and the Rayleigh numbers. In addition, values of $N u$ and $S h$ seem to be very similar but weaker for opposing situation compared to the aiding flow situation. This is in a good agreement with the flow patterns of thermal and solutal isocontours plotted in Fig. 5. It is worth mentioning that in such a case, the corresponding heat and mass transfer processes are ruled by pure diffusion solely $(N u=S h \approx 1)$.

Close scrutiny of the average Nusselt and Sherwood profiles demonstrates that the flow rates are minimum at the buoyancy ratio value $\mathrm{N}=-1$ for all Rayleigh numbers. It important to point out that, at this specific value of buoyancy ratio, the effects of thermal and solutal cells are of comparable magnitude as revealed by the 2-D numerical work of Nishimura et al. [1]. Moreover, a similar behavior of the flow rates presenting a minimum at $\mathrm{N}=-1$ was also observed by Sezai and Mohamad [3] for a cubic enclosure.

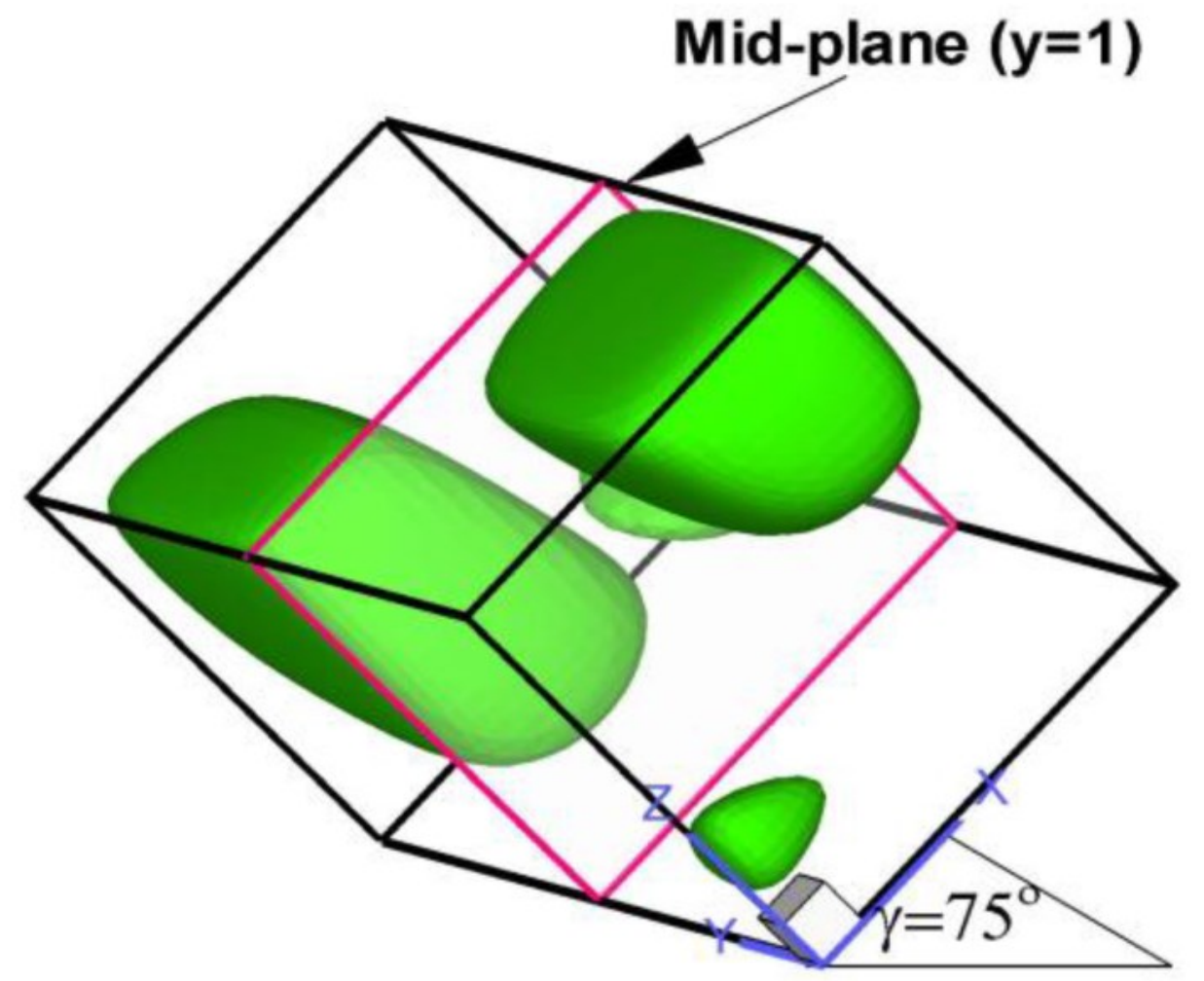

Fig. 7 Iso-surfaces of the transverse velocity component $v$ for the specific value $(0.00269)$ at $N=-1$ and $\mathrm{R}=2 \cdot 10^{4}$.

On another hand, and in order to demonstrate the three-dimensionality of the flow, the isosurfaces of the transverse v-velocity component is illustrated in Fig. 7 for the specific value $(0.00269)$ at $\mathrm{N}=-1$. As seen in this figure, the $3-\mathrm{D}$ structures presenting a dissymmetry with respect to the mid-plane ( $\mathrm{y}=1$ ) still manifest even for $\mathrm{N}=-1$ which corresponds to the value where $u$ and $S h$ are minimum. 


\section{CONLUDING REMARKS}

A numerical study of 3-D double diffusive natural convection in a solar distiller inclined distiller tilted at an angle $\gamma=75$ is performed in the current study. It is found, that despite the fact that the chosen angle inclination corresponds to a minimum of heat and mass transfer rates, the thermosolutal natural convection can be controlled by varying both parameters the Rayleigh and buoyancy ratio numbers. In fact, it is observed that, both $\mathrm{u}$ and w-velocity profiles norm increases with the Rayleigh number presenting a perfect symmetry about the centerlines of the cavity. Moreover, comparison of the isocontours relative to w-velocity components related to $\mathrm{Ra}=10^{3}$ and $2.10^{4}$ show the presence of two vortices which exhibit a great distortion and approach to the active walls by increasing $\mathrm{Ra}$ values. Meanwhile, by augmenting $\mathrm{Ra}$ values from $R a=10^{3}$ to $R a=2 \times 10^{4}$, iso-surfaces relative to temperature field undergoes a central stratification while the lower and upper gradients seem to be significantly strengthened. In terms of buoyancy ratio effects, thermal and concentration isocontours demonstrated that the flow intensity increases by gradually increasing $\mathrm{N}$ for aiding flow situation $(\mathrm{N}>0)$. Whereas, and for opposing flow case, heat and mass rates seem to have no significant effect by augmenting the buoyancy ratio but in contrast iso-contours are observed to be parallel and compressed at the vicinity of the active walls. This was observed to be in good agreement with the variation profiles of both average $N u$ and $S h$ numbers with respect to N. In fact, examination of the profile trends relative to opposing flow shows that average Nusselt and Sherwood values remain almost constant for $\mathrm{N}<-1$, while the corresponding profiles seem to undergo an augmenting behavior thereafter. In addition, and according to all Rayleigh number values, the variation of average Nusselt and Sherwood numbers is observed to be minimum for $\mathrm{N}=-1$. On another hand, isosurfaces of the transverse v-velocity component showed that 3-D effects still manifest even for the specific value $\mathrm{N}=-1$.

\section{References}

[1] T.N. Nishimura, M. Wakamatsu, and A.M. Morega, Oscillatory double-diffusive convection in a rectangular enclosure with combined horizontal temperature and concentration gradients. Int. J. Heat Mass Transfer, vol. 41, pp. 1601-1611, 1998.

[2] F. Oueslati, B. Ben-Beya and T. Lili, "Numerical investigation of thermosolutal natural convection in a rectangular enclosure of an aspect ratio four with heat and solute sources," Heat Mass Transfer, vol. 50, 721-736, 2014.

[3] I. Sezai, and A.A. Mohamad, "Double diffusive convection in a cubic enclosure with opposing temperature and concentration gradients," Physics of Fluids, vol. 12, pp. 2210-2223, 2000 .

[4] F. Oueslati, B. Ben Beya and T. Lili, "Numerical simulation of unsteady double-diffusive natural convection within an inclined parallelepipedic enclosure," Int. J. of Modern Physics C, vol. 25, pp. 1450058-1-25, 2014.

[5] K.K. Murugavel, P. Anburaj, R. S. Hanson, and T. Elango, "Progresses in inclined type solar stills", Renew. Sust. Energ. Rev., vol. 20, pp. 364-377, 2013.

[6] K. Sampathkumar, T.V. Arjunanb, P. Pitchandia, and P. Senthilkumarc, "Active solar distillation-a detailed review," Renewable and Sustainable Energy Reviews, vol. 14, pp. 15031526, 2010.

[7] N. Rahbar, J.A. Esfahani, "Productivity estimation of a single-slope solar still: Theoretical and numerical analysis," Energy, vol. 49,

[8] A. Madhlopa and C.M. Johnstone, "Computation of solar radiation distribution in a solar still withinternal and external reflectors. Solar Energy, vol. 85, pp. 217-233, 2011. 
[9] R. Chouikh, L. Ben Snoussi, and A. Guizani, "Numerical study of the heat and mass transfer in inclined glazing cavity, Application to a solar distillation cell," Renew. Energy, vol. 32, pp. 1511-1524, 2007.

[10] A. Omri, "Numerical investigation on optimization of a solar distiller dimensions," Desalination, vol. 206, pp. 373-379, 2007.

[11] M.M. Rahman, H. F.Öztop, A. Ahsan, M.A.Kalam, and Y. Varol, "Double-diffusive natural convection in a triangular solar collector," Int. Communications in Heat and Mass Transfer, vol. 39, pp. 264- 269, 2012.

[12] K. Ghachem, L. Kolsi, C. Maatki, A.K. Hussein and M.N. Borjini, "Numerical simulation of three-dimensional double diffusive free convection flow and irreversibility studies in a solar distiller," Int. Communications in Heat and Mass Transfer, vol. 39, pp. 869-876, 2012.

[13] B. Ben-Beya, and T. Lili, ” Three-dimensional incompressible flow in a two-sided non-facing lid-driven cubical cavity," C. R. Mecanique, vol. 336, pp. 863-872, 2008.

[14] D.L. Brown, R. Cortez, and M.L.Minion, "Accurate projection methods for the incompressible Navier-Stokes equations,” J. Comput. Phys., vol. 168, pp. 464-499, 2001.

[15] N. Ben-Cheikh, B. Ben-Beya, and T. Lili, "Benchmark solution for time-dependent natural convection flows with an accelerated full-multigrid method," Numerical Heat Transfer Part Bfundamentals, vol. 52, pp. 131-151, 2007.

[16] A. Hadjidimos, "Successive overrelaxation (SOR) and related methods," J. Comp. App. Math., vol.123 pp. 177-199, 2000. 